\title{
Hydrogen-Bonded Water Clusters Confined in Nanocavity as Bright Color Emitters
}

\author{
Tai-Qun Yang, ${ }^{*}$ Bo-Peng, ${ }^{*}$ Jia-Feng Zhou, ${ }^{*}$ Bing-Qian Shan, Kun Zhang \\ Shanghai Key Laboratory of Green Chemistry and Chemical Processes, Laboratory of Interface and \\ Water Science, College of Chemistry and Molecular Engineering, East China Normal University, \\ Shanghai 200062, China; \\ * Correspondence: kzhang@chem.ecnu.edu.cn (K.Z.)
}

\begin{abstract}
Molecules confined in the nanocavity and nanointerface exhibits rich of unique physicochemical properties, e.g., green fluorescent protein (GFP). However, the physical origin of their photoluminescence (PL) emission remains elusive. To mimic the microenvironment of GFP protein scaffold at the molecule level, two groups of nanocavities were created by molecule self-assembling using organic chromophores and by organic functionalization of mesoporous silica, respectively. We provide strong evidence that, the hydrogen-bonded water clusters (WCs) confined in these nanocavities are true color emitters with a universal formula of $\left\{X^{+} \cdot\left(\mathrm{OH}^{-} \cdot \mathrm{H}_{2} \mathrm{O}\right)\left(\mathrm{H}_{2} \mathrm{O}\right)_{n-1}\right\}$, in which $X$ is hydrated protons $\left(\mathrm{H}_{3} \mathrm{O}^{+}\right)$or protonated amino $\left(\mathrm{NH}_{3}{ }^{+}\right)$groups. Further controlled experiments and combined characterizations by time-resolved steady-state and ultra-fast transient optical spectroscopy showed that, the PL efficiency of WCs strongly depends on the type and stability of WCs structure, which is dominated by $\mathrm{H}$-Bonds. Differing from the local excitation of organic chromophores, the excited state of WCs has the characteristic of the topological excitations due to the many-body nuclear quantum effect (MBNQE) in confined nanocavity.
\end{abstract}

\section{Introduction}

Under ambient conditions, most surfaces are covered by water molecules. However, the influence of the adsorption of water molecules on the interfacial state at nanoscale interfaces was seriously overlooked in both chemistry and biology. Huge amount of experiment evidences prove that the first contact layer of water is not comprised of pure water but instead of a mixture of water and hydroxyl molecules, also called water clusters (WCs) in some cases.(1) It forms because they afford the optimal balance of $\mathrm{H}$-bonding within the overlayer and bonding to the surface,(2) which has now been observed on several transition metal oxide,(3) metal surfaces,(4) and even in biology system, such as green fluorescent protein (GFP). $(5,6)$ Intrinsically, free water molecules are colorless liquid. But, the pioneered $a b$ initio path-integral molecular dynamics (PIMD) simulations exhibits that, at confined nanocavity and interface, due to many-body nuclear quantum effects (MBNQEs), WCs could show many exotic quantum phenomena and their excited state is extremely susceptible to the structural inhomogeneity and local 
environments.(7-12)

The aggregation induced emission (AIE) effect was first proposed by Tang and his coworkers in 2001, which now has been widely concerned.(13, 14) The restriction of their intramolecular motions (RIM) in an aggregate state is the main cause of the AIE process. Thus, the AIE luminogens (AIEgens) with complicated chemical structure is necessary, such as diphenyl-1pyrenylphosphine (DPPP) with unique AIE rotors.(15-17) However, just by this mechanism, many abnormal photoluminescence (PL) properties of simple AlEgens with nonconjugated structures cannot be easily understood, such as tetraphenylethane (TPE) and 1,2-diphenylethane (s-DPE),(18-20) and even currently used 1-Bromopyrene (BP). It is importantly noted that, the nature of AIE is indeed a solvent-driven molecular self-assembly process, in which the poor solvent water is commonly used as mixed solvent. Considering the bizarre quantum effect of hydrogen-bonded water molecules in confined space, whether there is a hidden possibility that WCs in the cavity formed by AIE aggregate is the true luminous center,(21) instead of aggregate itself, which is also reminiscent of PL mechanism of GFP where structural water molecules in the proximity of GFP chromophore and several conserved amino-acid residues play the key role to tune the PL emission of GFP.(22-25) Our research provides direct evidence that WCs confined in AIE aggregates and silica matrix functionalized with simple amino $\left(-\mathrm{NH}_{2}\right)$ or carboxyl $(-\mathrm{COOH})$ groups could act as an emitter center to tune the PL emission with bright colors. Furthermore, the controlled experiments and combined characterizations by time-resolved steady-state and ultra-fast transient optical spectroscopy showed that, the PL efficiency of WCs is strongly dependent on the type and stability of WCs structures and the delicate interplays of $\mathrm{H}$-bonds in confined nanocavities.

\section{Results and discussions}

\section{Nanocavity formed by aggregation of typical AlEgens of diphenyl-1-pyrenylphosphine (DPPP) and tetraphenylethylene (TPE), and classical organic chromophores (1-Bromopyrene, BP) for WCs hosting}

Typical AIEgens of DPPP and TPE derivatives are deliberately selected to create the nanocavity by their self-assemblies. As previously reported, the as-synthesized DPPP aggregate exhibits strong solvophilicity-dependent photoluminescence emission features due to AIE effect,(15) as shown in Figure 1a and $\mathrm{c}$. When dissolved in ethanol solution, the DPPP molecule only exhibits three fingerprint emission peaks $\left(\lambda_{\max } \sim 380 \mathrm{~nm}, 400 \mathrm{~nm}\right.$ and $420 \mathrm{~nm}$ ) within the range of about $370-450 \mathrm{~nm}$, which corresponding to the $I \|$, IV and $I V$ characteristic emission peaks of pyrene, respectively. When DPPP was dispersed into the mixed solution of water and ethanol, with the increase of the volume fraction of water in the solvent $\left(R_{w}=V_{o} l_{w} / V_{0} l_{E}+w\right.$, the subscript $W$ and $\mathrm{E}$ represent water and ethanol, respectively), the PL of DPPP molecules gradually intensified when $R_{w}$ was increased to $70 \%$ and then suddenly dropped sharply due to $A C Q$ effect. When $R_{w}$ exceed a critical value $\left(R_{w} \geq\right.$ 
$70 \%$ ), a new broad bright emission emerged at $~ 515 \mathrm{~nm}$ with FWHM around $100 \mathrm{~nm}$ was intensified with the further increase of $R_{w}$, which is generally attributed to AIE effect (Fig. 1e, right, obvious Tyndall effect indicates the formation of aggregates).

(a)
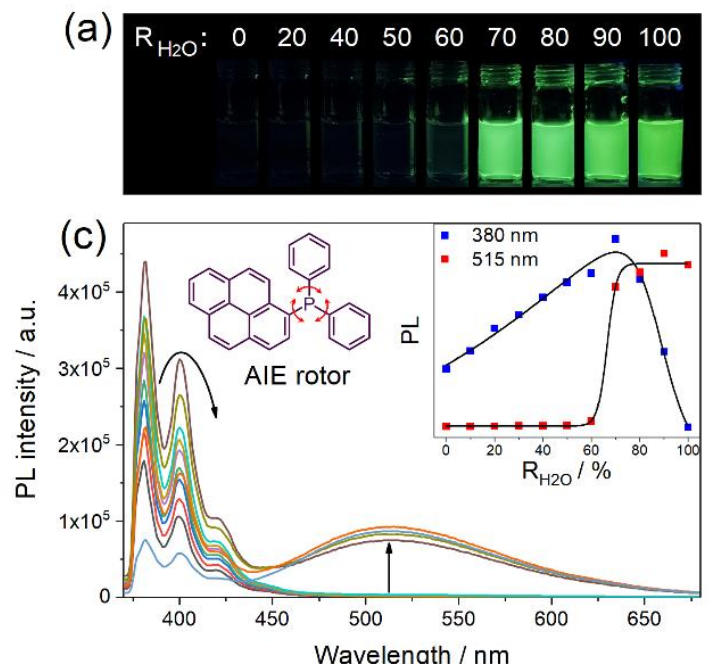

(e)

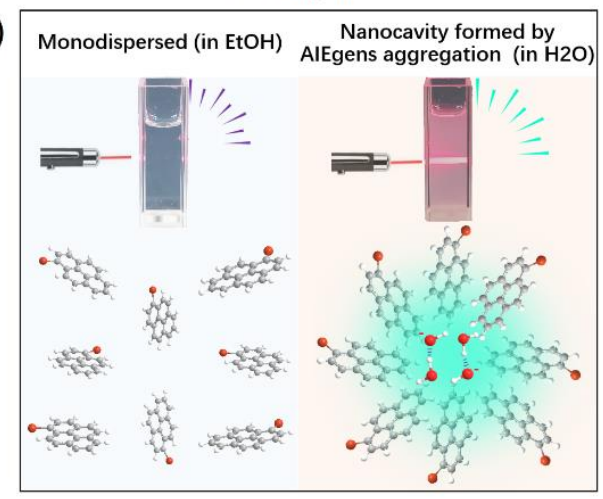

(b)
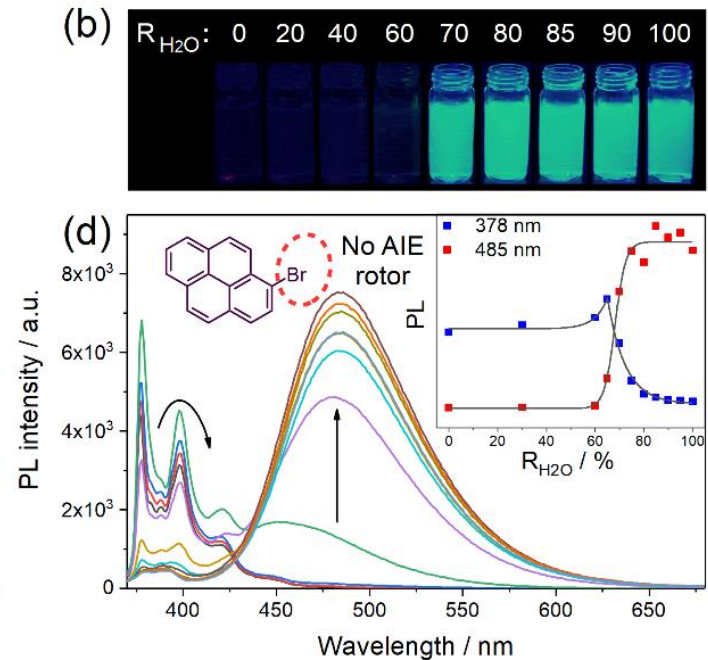

(f)

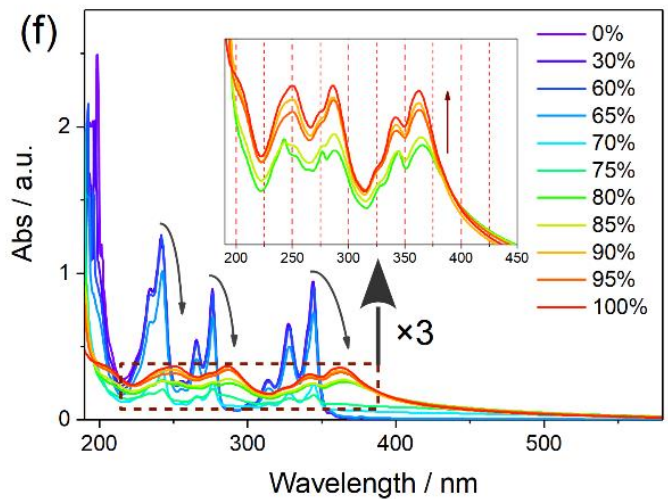

Figure 1. (a, b) Photographs and (c, d) fluorescence spectra of DPPP (a, c) and 1-bromopyrene $(b, d)$ in mixed solvents with different volume fraction $R_{w}$. Inset shows the relationship between the luminescence intensity and $R_{w}$. (e) Proposed mechanism for water induced AIE phenomenon. (f) Ultraviolet-visible (UV-vis) absorption spectra of 1-bromopyrene (BP) in mixed solvents with different $R_{w}$. All of the spectra were recorded immediately after the sample added into the mixture solvents; the concentration of DPPP and BP was 10 and $100 \mu \mathrm{mol} / \mathrm{L}$, respectively. Noting that every spectra acquisition need accomplish at a very short time (within 6 seconds) since DPPP are very sensitive to the UV light.

To our big surprises, in the same conditions, the conventional 1-bromopyrene (BP) chromophore used as a precursor for the synthesis of DPPP AIEgens (Scheme S1 and Figure S1)(26) exhibited the same water induced PL behaviors (Fig. 1b and 1d). The result confirms that the propeller-like rotor structure is not indispensable and implies an emission mechanism different with the classical AIE mechanism. Noting that both DPPP and BP aggregates in the mixed solvent exhibited similar emission properties, including a few percent emission quantum yield (QY) and several nanoseconds lifetime (Figure S5 and Table S1). The subtle difference of PL 
spectra between DPPP and BP is just a blue shift of emission at long wavelengths from $515 \mathrm{~nm}$ to $485 \mathrm{~nm}$. Very interestingly, the emission of BP molecules at $485 \mathrm{~nm}$ did not show the excitation-wavelength dependent red-shift behavior (Fig. S6), while the steady absorption of multi-bands shows a significant red-shift (Fig. 1f). This cannot be simply explained by AIE mechanism, concomitantly against the local excitation of conventional chromophores.
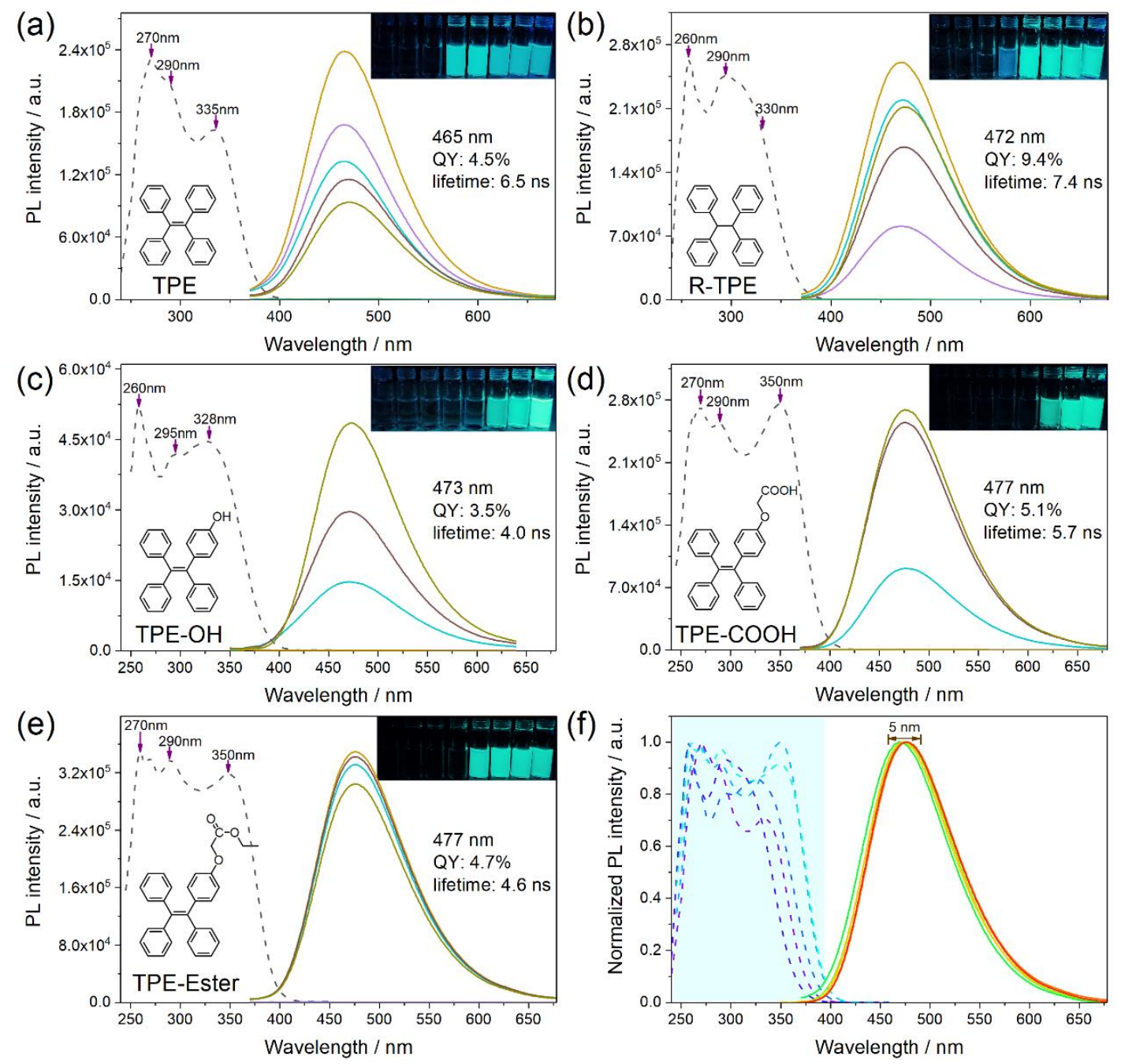

Figure 2. (a-e) Fluorescence spectra of five kinds of TPE derivatives in mixed solvents with different volume fraction ( $\mathrm{R}_{\mathrm{w}}: 0 \%, 20 \%, 40 \%, 60 \%, 70 \%, 80 \%, 90 \%, 100 \%$ ). Inset shows the corresponding digital photos under UV light. (f) Normalized fluorescence spectra of five kinds of TPE derivatives in water $\left(R_{w}=100 \%\right)$. The concentration of TPE derivatives was $100 \mu \mathrm{mol} / \mathrm{L}$.

Obviously, if the PL emission of AIEgens is coming from the aggregate itself, the absorption and emission spectra AlEgens with varied functional groups should be different. However, completely different TPE derivatives exhibits the same PL emission at ca. $480 \mathrm{~nm}$ with an identical multiple excitation bands in a range of 250-370 nm and similar fluorescence lifetime of ca. $5 \mathrm{~ns}$ (Fig. 2, scheme S2-S4, Fig. S2-S4, Fig. S7 and Table S2). In addition, we note that, even though TPE derivatives and BP have totally different chemical structure 
and conjugated structure, these two molecules at critical $R_{w}$ value of ca. $60 \%$ show an identical PL emission at ca. $480 \mathrm{~nm}$ (Fig. 1d and Fig. 2). Obviously, the PL origin of AlEgens is not from the aggregate itself. Whether some important clues are neglected? There is an overlooked fact that the nanocavity formed Water-AIEgens-Ethanol ternary system is an ideal platform to host the guest water molecules as PL emitters since all the hydrophobic organic chromophores at critical value of $R_{w}$ exhibits the presence of pre-Ouzo structure, i.e., the formation of milk-like micro-emulsions (Fig. S8 and S9). (27, 28) The dosing experiment of Hofmeister series ions and the isotope experiments of water tested the possibility of WCs as emitter centers. When various Hofmeister series ions were introduced, the wavelength of fluorescence emission was not shifted, but the emission intensity significantly increased, suggesting the increased stability of emitter center of WCs (Fig. $\mathrm{S} 10, \mathrm{a}$ and $\mathrm{b}$ ). The isotope experiments of water directly prove that the PL intensity of $\mathbf{B P}$ is strongly dependent on the $\mathrm{H}$-bonding capacity: stronger $\mathrm{H}$-Bonding interaction induces the more intense $\mathrm{PL}$ emission in order of $\mathrm{D}_{2} \mathrm{O}>$ $\mathrm{H}_{2}{ }^{18} \mathrm{O}>\mathrm{H}_{2} \mathrm{O}$ (Fig. S10c).(29) Thus, we tentatively assigned WCs confined in nanocavity as chromophores, completely differing from the conventional AIE mechanism. But, the precise structure of emitter center is difficult to be defined. Later we will discuss this intricate but important issue.

\section{Mesoporous nanocavity modified by targeted organic functions is full equivalent to the tertiary structure of native GFPs.}

Although we have identified WCs as emitters in the nanocavity of AlEgens, the conjugation of $\pi$ electrons in the organic chromophores by $\pi-\pi$ or $\pi-\mathrm{H}$ weak interactions to tune PL emissions cannot be completely precluded.(30) More importantly, the structure of WCs as emitter is not clear. Using mesoporous silica nanospheres (MSNs) with the spherical pores of ca. $3 \mathrm{~nm}$ as inorganic matrix, $(28,31)$ reminiscent of the size of $\beta$-can of $4.2 \mathrm{~nm}$ by 2.4 $\mathrm{nm}$ folded by eleven $\beta$-strands of GFP (Figure S11). The fluorescent MSNs (FMSNs) without the incorporation of chromophores were designed to show the central role of gusted WCs for the PL emission. To produce the adsorption sites of WCs in confined mesopores and copy the protein architecture of GFP, the silica was functionalized by customer-tailored organic functions. The selected organic functions should satisfy two requirements with non-conjugated structure and $\mathrm{H}$-Bonding. The very simple amino $\left(-\mathrm{NH}_{2}\right)$ and carboxyl $(-\mathrm{COOH})$ groups are used, since two conserved amino acid R96 and E222 residues in the nanocavity of GFP and GFP mutants were found to be very important to tune the PL properties.(5) With commonly used surface grafting technique,(32) the amino and carboxyl groups were successfully covalently tethered onto the surface of mesopores using aminopropyl triethoxysilane (or $\mathrm{N}$-(2-aminoethyl)-3-amino- propyltrimethoxysilane) and 3-(triethoxysilyl) propylsuccinic anhydride as coupling agent, respectively (Fig. 3, a and b, and Fig. S12). 

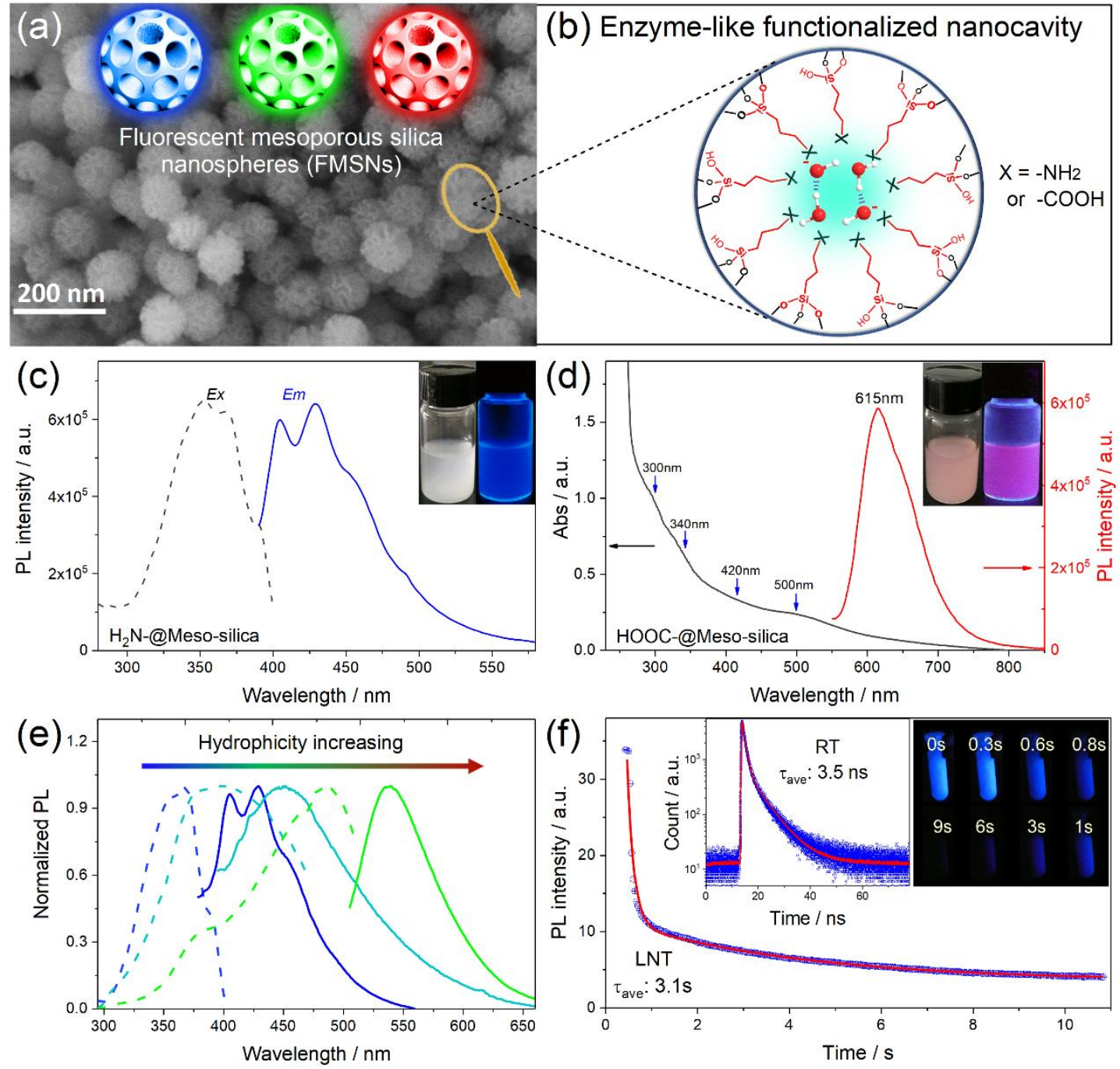

Figure 3. (a) Scanning electron microscopy (SEM) of as-synthesized fluorescent mesoporous silica nanoparticles (FMSNs). Inset shows the cartoon of FMSNs with different emission wavelength by surface modification under different conditions. (b) The formation of water clusters in amino- and carbonyl- groups functionalized enzyme-like nanocavity. (c) Excitation and emission spectra of aminopropyl-functionalized FMSNs. (d) Absorption and emission spectra of propylsuccinic-functionalized FMSNs. (e) Emission wavelength regulation of FMSNs by controlling the hydrophobicity of the nanopores. Normalized excitation (dashed) and emission (solid) spectra of amino functionalized DMSN ( $\mathrm{NH}_{2}-\mathrm{FMSNs}$ ) with hydrophobic treatment. The hydrophobic functional groups (trimethyl chlorosilane: TMCS) were gradually decreased from 4 equivalents (blue) to 1 equivalent (green) and the emission wavelength was red shifted from $\sim 430 \mathrm{~nm}$ to $\sim 538$ $\mathrm{nm}$. (f) Time-resolved luminescence decay profiles of $\mathrm{NH}_{2}-\mathrm{FMSNs}$ at room temperature (RT) and liquid nitrogen temperature (LNT). Inset shows the naked eye visible long afterglow emission of $\mathrm{NH}_{2}$-FMSNs at LNT.

It is important to note that both individual amino and succinic functional groups are non-luminescent in solution. However, if they are clustered in the pores of MSNs, MSNs emit very strong PL luminescence with tunable colors, and the absorption and emission bands strongly depended on the grafted 
molecules (Fig. 3c and 3d). The amino-functionalized MSNs showed a striking blue-light fluorescence emission at ca. $430 \mathrm{~nm}$, and its excitation and emission spectra completely matched, implying a nonconventional molecule local excitation (Fig. 3c) since the chromophores are absent in FMSNs. While, the succinic functionalized MSNs exhibited a remarkable red-color emission at ca. $615 \mathrm{~nm}$ (Fig. 3d). Time resolved PL spectra showed that both samples have short lifetimes of several ns and moderate quantum yield ( 1.0\%). Generally, the excited transition of a single amino and carbonyl group in a range of 300$500 \mathrm{~nm}$ cannot be distinguished in the UV adsorption spectrum. However, due to the many-body nuclear quantum effects (MBNQE) among the $\mathrm{H}$-bonded WCs in the confined nanocavity of MSNs, some new quantum states emerge through the space overlapping of the $\mathrm{p}$ orbitals of $\mathrm{O}$ atoms in neighboring water molecules, $(33,34)$ whose intense and multiple adsorption bands have $\pi-\pi^{*}$ transition characteristics and excitation bands have a feature of topological excitation. In our previously published results, these intermediate states were called $p$-band emitter centers.(33) If the production of these new states is due to the overlapping of $p$ orbitals from neighboring $O$ atoms, the stability of new formed quantum states will be strongly fluctuated by $\mathrm{H}$-bond interactions from the surrounding environments, consequently exhibiting different PL properties.

As expected, when the surface hydrophobicity was precisely tuned, FMSNs show the distinct PL emissions. With the increase of hydrophobicity of $\mathrm{NH}_{2}$-FMSNs, the blue emission at ca. $430 \mathrm{~nm}$ was red-shifted to $530 \mathrm{~nm}$, concomitantly accompanying a gradual red-shift of excitation wavelengths (Fig. 3e). This indicates that the delicate change of microenvironment significantly influences the quantum state of WCs in the nanocavity, which is reminiscent of spectral and photochemical properties of an irreversible photoinduced GFPs based upon oxidative decarboxylation of reserved Glu 222 residue.(35) Most interestingly, when the FMSNs were cooled down to liquid nitrogen temperature $(77 \mathrm{~K})$, the ultra-long quantum lifetime up to ten seconds was observed from c.a. $3.5 \mathrm{~ns}$ with eight order of magnitude increase (Fig. 3f). The increase of quantum lifetime is probably due to the slowing down of proton shuttling in the nanocavity at low temperature, which stabilized the dynamic of excited states. Our early reported electron paramagnetic resonance (EPR) results showed that, the fast switching of hydroxide $\left(\mathrm{OH}^{-}\right)$to water in the mesopores on the ns scale was almost suspended at the liquid nitrogen temperature (LNT) due to the blocking of proton transfer.(32) The results of PL performance at LNT suggest that the WCs in the nanocavity is probably present in the form of $\left\{\mathrm{NH}_{3}{ }^{+} \cdot\left(\mathrm{OH}^{-} \cdot \mathrm{H}_{2} \mathrm{O}\right)\left(\mathrm{H}_{2} \mathrm{O}\right)_{n-1}\right\}$, where protonated amine is adsorbing sites for a singly hydrated hydroxyl complex $\left(\mathrm{OH}^{-} \cdot \mathrm{H}_{2} \mathrm{O}\right)$ and concomitantly as charge compensators. Water molecules in the WCs are presented in symmetric and asymmetric hydrogen bond (the latter is normal $\mathrm{H}$-bond). The symmetric hydrogen bond $(\mathrm{HO}-\mathrm{H}-\mathrm{OH})$ in a water-hydroxyl complex has high stability, where the protons become delocalized between the 
$\mathrm{O}$ nuclei with very short $\mathrm{O}-\mathrm{O}$ separations, leading to the formation of new multiple quantum states. $(1,2,4)$ Recently, it was actually observed by X-ray diffraction in compressed ice, wherein the $\mathrm{O}-\mathrm{O}$ distance reached $2.4 \AA$ under high pressure of $60 \mathrm{GPa}$.(36-38) To simply the structure of WCs, the formula can also be written as $\left\{\mathrm{NH}_{3}{ }^{+} \cdot\left(\mathrm{H}_{3} \mathrm{O}_{2}\right)^{-}\left(\mathrm{H}_{2} \mathrm{O}\right)_{n-1}\right\}$. Thus, the stability of hydrated hydroxyl complex, in other words, the PL efficiency of emitter centers, strongly depends on the synergetic interactions among adsorption site $\left(-\mathrm{NH}_{3}+\right)$, hydrated hydroxyl complexes $\left(\mathrm{H}_{3} \mathrm{O}_{2}-\right)$ and surrounding water molecules. Our acid titration and ion dosing experiments further prove this hypothesis (Fig. 4).
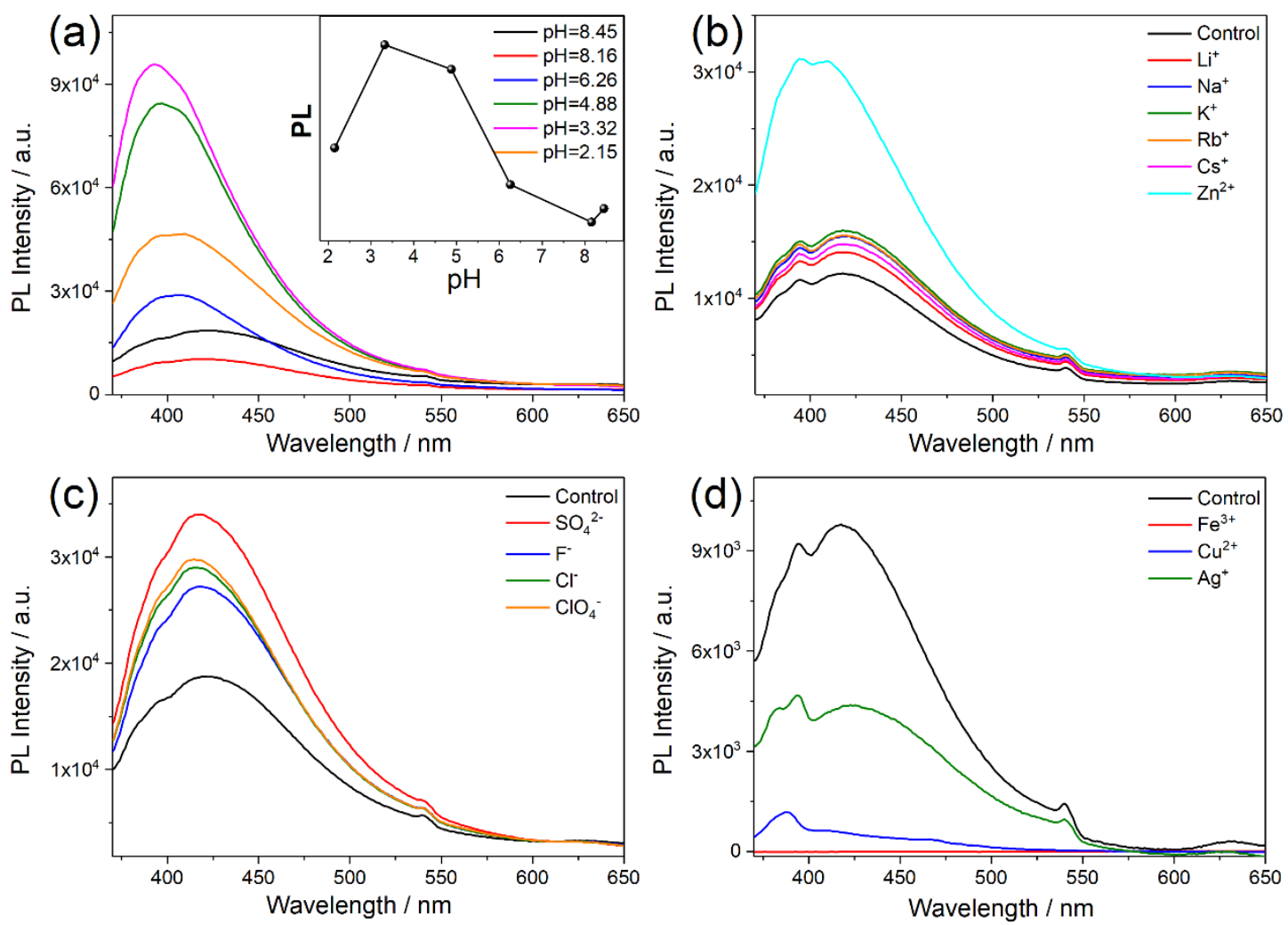

Figure 4. (a) Emission spectra of $\mathrm{NH}_{2}-\mathrm{FMSNs}$ in water solution with different $\mathrm{pH}$ values. Emission spectra of $\mathrm{NH}_{2}$-FMSNs with different cations (b, d) and anions (c). The concentration of ions was $5 \mathrm{mmol} / \mathrm{L}$. All spectra are obtained with $350 \mathrm{~nm}$ excitation.

With the increase of $\mathrm{pH}$ value, an interesting evolution of FMSNs PL intensity was observed: At first, the intensity reduced by half, then gradually increased to the maximum value with a decrease of $\mathrm{pH}$, and finally decreased slightly again (Fig. 4 a). Considering the $\left\{\mathrm{NH}_{3}{ }^{+} \cdot\left(\mathrm{OH}^{-} \cdot \mathrm{H}_{2} \mathrm{O}\right)\left(\mathrm{H}_{2} \mathrm{O}\right)_{\mathrm{n}-1}\right\}$ structure of emitter center, the initial decrease of intensity can be readily understood due to the breakage of hydrated hydroxyl complexes by an acid-base neutralization reaction. The recovery of $\mathrm{PL}$ with the decrease of $\mathrm{pH}$ indicates the formation of a new luminescent species, which has a similar structure with that in the base conditions, since the emission wavelength at $430 \mathrm{~nm}$ was not changed in the acid conditions $(\mathrm{pH}<7.0)$. Thus, a new structures with the formula of $\left\{\mathrm{H}_{3} \mathrm{O}^{+}\left(\mathrm{OH}^{-} \cdot \mathrm{H}_{2} \mathrm{O}\right)\left(\mathrm{H}_{2} \mathrm{O}\right)_{n-1}\right\}$ was assigned where a protonated water acts as an adsorption site. Since the solution of AlEgens and carboxylate functionalized 
FMSNs is also acidic $(\mathrm{pH}<7.0)$, the hydrated proton riveted WCs should be the main emission center. Overall, acid titration demonstrates the pivotal role of hydrated hydroxyl complex in the emitter center to tune the PL. The observed interesting shifts between two structures probably answers why, in both acid and base condition, optical and electro-catalysts for small molecule activations $\left(\mathrm{CO}_{2}, \mathrm{CO}, \mathrm{H}_{2} \mathrm{O}, \mathrm{N}_{2}\right.$ and $\mathrm{O}_{2}$, etc.) exhibit the excellent chemical reactivity, which is independent on the nature of active sites with metal or nonmetals (such as carbon or heteroatoms doped carbon materials).(39-41)

Due to its super-stability of $\mathrm{H}$-bond hydrated hydroxyl complex, the dosing of Hofmeister series ions slightly perturbs the interaction between hydrated hydroxyl complex and surrounding water molecules. Owing to the salt-out effect of Hofmeister series ions, the weak $\mathrm{H}$-bonded water molecules near the hydrated hydroxyl complex are removed, leading to the improvement of hydrated hydroxyl complex's stability. Consequently, PL emission of FMSNs in figure $4 \mathrm{~b}$ and $4 \mathrm{c}$ was intensified. Importantly note that, $\mathrm{PL}$ of FMSNs is extremely sensitive to $\mathrm{Zn}^{2+}$ cations (Fig. $4 \mathrm{~b}$ ), as observed in GFP. A zinc finger domain has been inserted in place of Tyr145 in EYFP, which increased fluorescence about 1.7 times without a change in wavelength.(26) However, the addition of transition metal cations $\left(\mathrm{Fe}^{3+} / \mathrm{Cu}^{2+} / \mathrm{Ag}^{+}\right)$, due to the competing adsorption on " $\mathrm{H}_{3} \mathrm{O}_{2}$ " " anionic complexes between metal cations and protonated amino groups $\left(-\mathrm{NH}_{3}{ }^{+}\right)$, the structure of stabilized emitters was broken, consequently diminishing PL emission dramatically (Fig. 4 d), which is again a reminiscent of fact that metal ions in the vicinity of a chromophore of GFP are known to quench fluorescence in a distance-dependent fashion.(42) Taken all together, the PL emission of AlEgens and FMSNs is a result of synergetic interaction of three main components in the emitter center. Based on this discovery, we deduce that, the emitter center of GFP is not p-hydroxybenzylidene-imidazolidinone ( $\mathrm{HBDI}-\mathrm{OH}$ ) chromophore formed from internal cyclisation of Ser65, Tyr66 and Gly67, but water clusters (WCs) confined in in the center of the $\beta$-barrel of GFP, where the anionic HBDI-Ochromophore may play a hydroxyl role to form the $\{(\mathrm{HBDI}-\mathrm{O} W 46)(\equiv \mathrm{R}\}$ emitter center (in formula, W46 means $\mathrm{H}$-boned water with HBDI, and $\equiv \mathrm{R}$ mean the conserved amino-acid residues in the proximity of chromophore (Fig S10).(5) In the unique microenvironment of $\beta$-barrel, the structure of HBDI-O-W46 complex is extremely stabilized due to the strong $\mathrm{H}$-bond interactions with the conserved amino-acid resides, that is why GFP is one of the most stabilized nontoxic PL chromophores. 

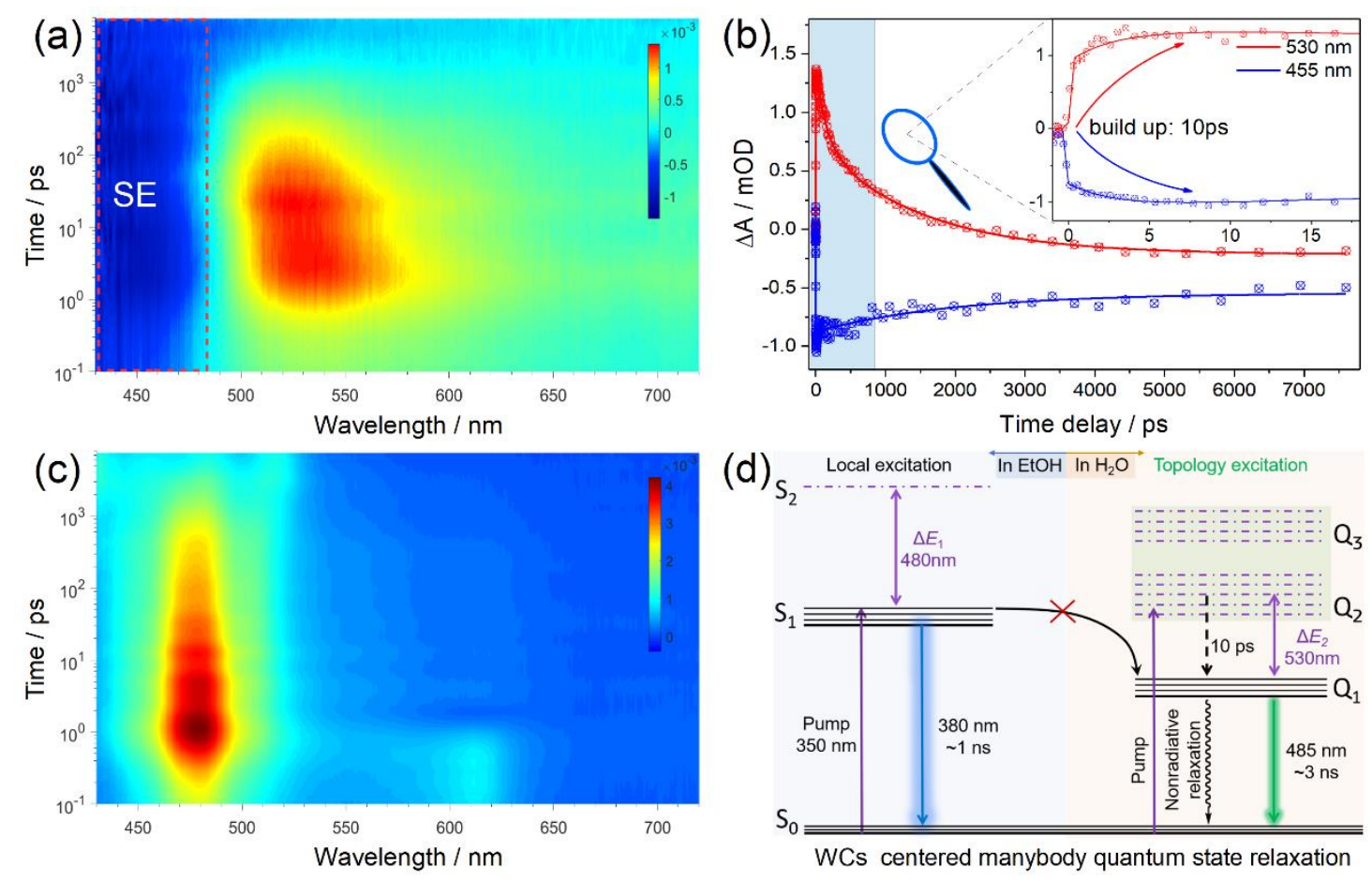

Figure 5. Contour representation of the multidimensional time-resolved transient absorption (TA) data for $\mathbf{B P}$ in $\mathrm{H}_{2} \mathrm{O}$ (a) and $\mathrm{EtOH}$ (c) with an excitation of $350 \mathrm{~nm}$. (b) Selected decay traces at $455 \mathrm{~nm}$ and $530 \mathrm{~nm}$ and corresponding fitting of BP in water solution; the fitting results are summarized in Table S4. (d) Energy levels, relaxation pathway of WCs under different solvent conditions $\left(Q_{n}\right.$ means the multiple quantum states, which is produced by many-body nuclear quantum effect).

\section{Kinetics of excited states of WCs in the confined nanocavity.}

To understand the dynamics of excited states of WCs in the confined nanocavity, the femtosecond time-resolved transient absorption (TA) spectra for BP AlEgens in solvent of $\mathrm{H}_{2} \mathrm{O}$ and $\mathrm{EtOH}$ with an excitation of $350 \mathrm{~nm}$ was performed (Fig. 5 a and $5 \mathrm{C}$ ). As expected, a transient absorption band of BP molecules centered at $475 \mathrm{~nm}$ in ethanol was observed, which is assigned to the excited state absorption (ESA) of BP molecule itself. When BP molecules were dispersed into water solution, two broad signals centered at $455 \mathrm{~nm}$ and $530 \mathrm{~nm}$ were captured, which was assigned to the stimulated emission (SE) due to the PL emission at $485 \mathrm{~nm}$ (Fig. 1d) and the excited state absorption of WCs in the aggregated nanocavity, respectively. The loss of excitation of BP molecule itself at $475 \mathrm{~nm}$ was due to ACQ effect. In addition, with a monoexponential decay fitting model, the kinetic traces of picosecond TA exhibit that the buildup process at $455 \mathrm{~nm}$ completely matches the ultrafast decay at 530 (Fig. 5b), suggesting that the TA absorption band centered at 530 $\mathrm{nm}$ is the excited state of WCs PL. But, the relaxation route of excited electron is not clear, electron transfer between multiple quantum states $\left(Q_{n}\right)$ formed WCs (Fig. 5d, right in light-yellow background) or Förster energy transfer 
process of BP molecule to WCs (Fig. 5d, Left in light-blue background)? The fitting data of selected decay traces showed that the excited electron transfer just happens in 10 ps timespans and the excited state absorption signal of BP was vanished. (Fig. 5a) Based on these results, the electron transfer from BP molecules to WCs which happened in a relative long timescale could be precluded. (Fig. 5d, Black arrow marks the directions). The production of excited states $\left(Q_{n}\right)$ of WCs is a result of many-body nuclear quantum effect, coincided with the unique feature of steady-state absorption and excitation with multibands (Fig. 2a-e, Fig. $3 c$ and d, and S6). However, due to the sensitivity of $\mathrm{H}$-bond on the environment, very fine spectral peaks of adsorption band on the TA spectra cannot be observed, consequently only exhibited as a very broad peak in a range of $470-650 \mathrm{~nm}$. The observation of ultra-long quantum lifetime of FMSNs up to ten seconds at liquid nitrogen temperature indicates a nonconventional local excitation, herein we call it topological excitation. Thus, using $\left\{\mathrm{X}^{+} \cdot\left(\mathrm{OH}^{-} \cdot \mathrm{H}_{2} \mathrm{O}\right)\left(\mathrm{H}_{2} \mathrm{O}\right)_{\mathrm{n}-1}\right\}$ or $\left\{\mathrm{X}^{+} \cdot\left(\mathrm{H}_{3} \mathrm{O}_{2}\right)^{-}\left(\mathrm{H}_{2} \mathrm{O}\right)_{n-1}\right\}$ as model system, wherein $\mathrm{X}=\mathrm{NH}_{3}, \mathrm{H}_{3} \mathrm{O}$ or metals, by ab initio path-integral molecular dynamics (PIMD) simulation, $(7)$ the precise and fine quantum states could be simulated in the near future.

\section{Conclusion}

Based on the solid experimental evidences, we first demonstrated that well $\mathrm{H}$-Bonded $\mathrm{WCs}$ in the confined nanocavity is an effective emitter center, whose structure has a universal formula of $\left\{\mathrm{X}^{+\cdot} \cdot\left(\mathrm{OH}^{-} \cdot \mathrm{H}_{2} \mathrm{O}\right)\left(\mathrm{H}_{2} \mathrm{O}\right)_{\mathrm{n}-1}\right\}$. The strong $\mathrm{H}$-Bond $\mathrm{OH}^{-} \cdot \mathrm{H}_{2} \mathrm{O}$ complex in WCs was accurately adsorbed on the $X$ sites by electrostatic interaction, and its $\mathrm{H}$-Bond strength was strongly tuned by surrounding water molecules. The PL efficiency of emitters strongly depends on the nature and stability of the complex structure, and the hydrogen bond interaction with the surrounding water molecules. The origin of WC's PL was tentatively assigned to the many-body nuclear quantum effect (MBNQE) in the confined nanocavity. The proposed model not only elucidates the nature of AIE effect in chemistry, but also answers the photophysics and photochemistry of GFP in biology system.(43) Most importantly, considering the universality of water adsorption at any interfaces, the PL mechanism of all the quantum nanodots, including the metal nanoclusters $(\mathrm{NCs}),(44,45)$ carbon dot, $(46)$ MOF,(47) metal cation exchanged zeolites, $(48,49)$ supermolecules,(50-52) non-traditional chromophores without conjugated structures, $(53,54)$ perovskites, $(55,56)$ and even semiconductor, $(57)$ should be revisited.

\footnotetext{
Author Contributions: TQY, BP and JFZ equally contribute to this research. JFZ synthesize AIEgens and its derivatives. BP prepare and characterize the FMSNs. TQY performed the PL and TA measurements. BQS performed the SEM and TEM measurements. KZ conceived and directed the project. KZ-and TQY co-designed the figures and wrote the manuscript. All authors have read and agreed to the published version of the manuscript.
}

Acknowledgments: This research was funded by the NSFC (21872053 and 21573074), the Science and Technology Commission of Shanghai Municipality (19520711400), the CAS key laboratory of Low-Coal Conversion Science \& Engineering (KLLCCSE-201702), and the JORISS program, the Postdoctoral 
Science Foundation of China (2018M640360). K.Z. thanks ENS de Lyon for a temporary position as an invited professor in France. We also appreciate Prof. Shuanhu GAO for their great helps in the synthesis of AIEgens at East China Normal University.

\section{Reference}

1. M. Meiera, J. Hulva, Z. Jakub, J. Pavelec, M. Setvin, R. Bliem, M. Schmid, U. Diebold,

C. Franchini, G. S. Parkinson, Water agglomerates on Fe304(001). Proceedings of the National Academy of Sciences of the United States of America 115, 5642-5650 (2018).

2. X.-Z. Li, M. I. J. Probert, A. Alavi, A. Michaelides, Quantum Nature of the Proton in Water-Hydroxyl Overlayers on Metal Surfaces. Physical Review Letters 104, 066102 (2010).

3. W. Yuan, B. Zhu, X.-Y. Li, T. W. Hansen, Y. Ou, K. Fang, H. Yang, Z. Zhang, J. B. Wagner, Y. Gao, Y. Wang, Visualizing $\mathrm{H} 2 \mathrm{O}$ molecules reacting at $\mathrm{TiO} 2$ active sites with transmission electron microscopy. Science 367, 428-430 (2020).

4. T. Kumagai, M. Kaizu, H. Okuyama, S. Hatta, T. Aruga, I. Hamada, Y. Morikawa, Symmetric hydrogen bond in a water-hydroxyl complex on Cu(110). Physical Review B 81, (2010)10.1103/PhysRevB.81.045402).

5. T. D. Craggs, Green fluorescent protein: structure, folding and chromophore maturation. Chemical Society reviews 38, 2865-2875 (2009); published online EpubOct (10.1039/b903641p).

6. G. D. Scholes, G. R. Fleming, L. X. Chen, A. Aspuru-Guzik, A. Buchleitner, D. F. Coker, G. S. Engel, R. van Grondelle, A. Ishizaki, D. M. Jonas, J. S. Lundeen, J. K. McCusker, S. Mukamel, J. P. Ogilvie, A. Olaya-Castro, M. A. Ratner, F. C. Spano, K. B. Whaley, X. Zhu, Using coherence to enhance function in chemical and biophysical 
systems. Nature 543, 647-656 (2017); published online EpubMar 29 (10.1038/nature21425).

7. J. Guo, X.-Z. Li, J. Peng, E.-G. Wang, Y. Jiang, Atomic-scale investigation of nuclear quantum effects of surface water: Experiments and theory. Progress in Surface Science 92, 203-239 (2017)10.1016/j.progsurf.2017.11.001).

8. M. E. Tuckerman, D. Marx, M. L. Klein, M. Parrinello, On the Quantum Nature of the Shared Proton in Hydrogen Bonds. Science 275, 817-820 (1997).

9. J. Peng, D. Cao, Z. He, J. Guo, P. Hapala, R. Ma, B. Cheng, J. Chen, W. J. Xie, X. Z. Li, P. Jelinek, L. M. Xu, Y. Q. Gao, E. G. Wang, Y. Jiang, The effect of hydration number on the interfacial transport of sodium ions. Nature 557, 701-705 (2018); published online EpubMay (10.1038/s41586-018-0122-2).

10. X. Meng, J. Guo, J. Peng, J. Chen, Z. Wang, J.-R. Shi, X.-Z. Li, E.-G. Wang, Y. Jiang, Direct visualization of concerted proton tunnelling in a water nanocluster. Nature Physics 11, 235-239 (2015)10.1038/nphys3225).

11. J. O. Richardson, C. Pérez, S. Lobsiger, A. A. Reid, B. Temelso, G. C. Shields, Z. Kisiel, D. J. Wales, B. H. Pate, S. C. Althorpe, Concerted hydrogen-bond breaking by quantum tunneling in the water hexamer prism. Science $351,1310-1313$ (2016).

12. J. Guo, J.-T. Lü, Y. Feng, J. Chen, J. Peng, Z. Lin, X. Meng, Z. Wang, X.-Z. Li, E.-G. Wang, Y. Jiang, Nuclear quantum effects of hydrogen bonds probed by tip-enhanced inelastic electron tunneling. Science 352, (2016).

13. J. Luo, Z. Xie, J. W. Lam, L. Cheng, H. Chen, C. Qiu, H. S. Kwok, X. Zhan, Y. Liu, D. Zhu, B. Z. Tang, Aggregation-induced emission of 
1-methyl-1,2,3,4,5-pentaphenylsilole. Chemical communications, 1740-1741 (2001); published online EpubSep 21 (10.1039/b105159h).

14. H. Zhang, Z. Zhao, P. R. McGonigal, R. Ye, S. Liu, J. W. Y. Lam, R. T. K. Kwok, W. Z. Yuan, J. Xie, A. L. Rogach, B. Z. Tang, Clusterization-triggered emission: Uncommon luminescence from common materials. Materials Today 32, 275-292 (2020)10.1016/j.mattod.2019.08.010).

15. C. Xing, J. Liu, F. Chen, Y. Li, C. Lv, Q. Peng, H. Hou, K. Li, Diphenyl-1-pyrenylphosphine: photo-triggered AIE/ACQ transition with remarkable third-order nonlinear optical signal change. Chemical communications 56, 4220-4223 (2020); published online EpubApr 14 (10.1039/d0cc01031f).

16. Y. J. Kong, Z. P. Yan, S. Li, H. F. Su, K. Li, Y. X. Zheng, S. Q. Zang, Photoresponsive Propeller-like Chiral AIE Copper(I) Clusters. Angewandte Chemie 59, 5336-5340 (2020); published online EpubMar 23 (10.1002/anie.201915844).

17. X. Wu, C. Y. Huang, D. G. Chen, D. Liu, C. Wu, K. J. Chou, B. Zhang, Y. Wang, Y. Liu, E. Y. Li, W. Zhu, P. T. Chou, Exploiting racemism enhanced organic room-temperature phosphorescence to demonstrate Wallach's rule in the lighting chiral chromophores. Nature communications 11, 2145 (2020); published online EpubMay 1 (10.1038/s41467-020-15976-5).

18. H. Zhang, X. Zheng, N. Xie, Z. He, J. Liu, N. L. C. Leung, Y. Niu, X. Huang, K. S. Wong, R. T. K. Kwok, H. H. Y. Sung, I. D. Williams, A. Qin, J. W. Y. Lam, B. Z. Tang, Why Do Simple Molecules with "Isolated" Phenyl Rings Emit Visible Light? Journal of the American Chemical Society 139, 16264-16272 (2017); published online EpubNov 
$15(10.1021 / j a c s .7 b 08592)$.

19. H. Zhang, L. Du, L. Wang, J. Liu, Q. Wan, R. T. K. Kwok, J. W. Y. Lam, D. L. Phillips, B. Z. Tang, Visualization and Manipulation of Molecular Motion in the Solid State through Photoinduced Clusteroluminescence. The journal of physical chemistry letters 10, 7077-7085 (2019); published online EpubNov 21 (10.1021/acs.jpclett.9b02752).

20. Q. Li, Z. Li, The Strong Light-Emission Materials in the Aggregated State: What Happens from a Single Molecule to the Collective Group. Advanced science 4, 1600484 (2017); published online EpubJul (10.1002/advs.201600484).

21. S. Vaitheeswaran, H. Yin, J. C. Rasaiah, G. Hummer, Water clusters in nonpolar cavities. Proceedings of the National Academy of Sciences of the United States of America 101, 17002-17005 (2004); published online EpubDec 7 (10.1073/pnas.0407968101).

22. M. Zimmer, GFP: from jellyfish to the Nobel prize and beyond. Chemical Society reviews 38, 2823-2832 (2009).

23. R. Y. Tsien, The Green Fluorescent Protein. Annu. Rev. Biochem. 67, 509-544 (1998).

24. J. Dong, K. M. Solntsev, L. M. Tolbert, Activation and Tuning of Green Fluorescent Protein Chromophore Emission by Alkyl Substituent-Mediated Crystal Packing. J. Am. Chem. Soc. 131, 662-670 (2009).

25. S. L. Tou, G. J. Huang, P. C. Chen, H. T. Chang, J. Y. Tsai, J. S. Yang, Aggregation-induced emission of GFP-like chromophores via exclusion of solvent-solute hydrogen bonding. Chemical communications 50, 620-622 (2014); published online EpubJan 18 (10.1039/c3cc47262k). 
26. G. S. Baird, D. A. Zacharias, R. Y. Tsien, Circular permutation and receptor insertion within green fluorescent proteins. Proceedings of the National Academy of Sciences of the United States of America 96, 11241-11246 (1999).

27. T. N. Zemb, M. Klossek, T. Lopiana, J. Marcus, S. Schöettl, D. Horinek, S. F. Prevost, D. Touraud, O. Diat, S. Marčelja, W. Kunza, How to explain microemulsions formed by solvent mixtures without conventional surfactants. Proceedings of the National Academy of Sciences of the United States of America 113, 4260-4265 (2016).

28. P. Hao, B. Peng, B.-Q. Shan, T.-Q. Yang, K. Zhang, Comprehensive understanding of the synthesis and formation mechanism of dendritic mesoporous silica nanospheres. Nanoscale Advances 2, 1792-1810 (2020)10.1039/d0na00219d).

29. A. Berger, G. Ciardi, D. Sidler, P. Hamm, A. Shalit, Impact of nuclear quantum effects on the structural inhomogeneity of liquid water. Proceedings of the National Academy of Sciences of the United States of America 116, 2458-2463 (2019); published online EpubFeb 12 (10.1073/pnas.1818182116).

30. Y. Yang, X. Yang, X. Fang, K. Z. Wang, D. Yan, Reversible Mechanochromic Delayed Fluorescence in 2D Metal-Organic Micro/Nanosheets: Switching Singlet-Triplet States through Transformation between Exciplex and Excimer. Advanced science 5, 1801187 (2018); published online EpubNov (10.1002/advs.201801187).

31. K. Zhang, L. L. Xu, J. G. Jiang, N. Calin, K. F. Lam, S. J. Zhang, H. H. Wu, G. D. Wu, B. Albela, L. Bonneviot, P. Wu, Facile large-scale synthesis of monodisperse mesoporous silica nanospheres with tunable pore structure. Journal of the American Chemical Society 135, 2427-2430 (2013); published online EpubFeb 20 
(10.1021/ja3116873).

32. K. Zhang, T. Q. Yang, B. Q. Shan, P. C. Liu, B. Peng, Q. S. Xue, E. H. Yuan, P. Wu, B. Albela, L. Bonneviot, Dendritic and Core-Shell-Corona Mesoporous Sister Nanospheres from Polymer-Surfactant-Silica Self-Entanglement. Chemistry 24, 478-486 (2018); published online EpubJan 9 (10.1002/chem.201704714).

33. T. Yang, B. Shan, F. Huang, S. Yang, B. Peng, E. Yuan, P. Wu, K. Zhang, P band intermediate state (PBIS) tailors photoluminescence emission at confined nanoscale interface. Communications Chemistry 2, (2019)10.1038/s42004-019-0233-1).

34. R. Hoffmann, Interaction of orbitals through space and through bonds. Accounts of Chemical Research 4, 1-9 (2002)10.1021/ar50037a001).

35. V. Sample, R. H. Newman, J. Zhang, The structure and function of fluorescent proteins. Chemical Society reviews 38, 2852-2864 (2009); published online EpubOct $(10.1039 / \mathrm{b} 913033 \mathrm{k})$

36. M. Benoit, D. Marx, M. Parrinello, Tunnelling and zero-point motion in high-pressure ice. Nature 392, 258-261 (1998).

37. E. Schwegler, M. Sharma, F. Gygi, G. Galli, Melting of ice under pressure. Proceedings of the National Academy of Sciences of the United States of America 105, 14779-14783 (2008); published online EpubSep 30 (10.1073/pnas.0808137105).

38. J. A. Morrone, L. Lin, R. Car, Tunneling and delocalization effects in hydrogen bonded systems: A study in position and momentum space. J. Chem. Phys. 130, 204511 (2009)10.1063/1.3142828).

39. Y.-C. Hao, Y. Guo, L.-W. Chen, M. Shu, X.-Y. Wang, T.-A. Bu, W.-Y. Gao, N. Zhang, 
X. Su, X. Feng, J.-W. Zhou, B. Wang, C.-W. Hu, A.-X. Yin, R. Si, Y.-W. Zhang, C.-H. Yan, Promoting nitrogen electroreduction to ammonia with bismuth nanocrystals and potassium cations in water. Nature Catalysis 2, 448-456 (2019)10.1038/s41929-019-0241-7).

40. X. Wang, C. Xu, M. Jaroniec, Y. Zheng, S. Z. Qiao, Anomalous hydrogen evolution behavior in high-pH environment induced by locally generated hydronium ions. Nature communications 10, 4876 (2019); published online EpubOct 25 (10.1038/s41467-019-12773-7).

41. E. Liu, J. Li, L. Jiao, H. T. T. Doan, Z. Liu, Z. Zhao, Y. Huang, K. M. Abraham, S. Mukerjee, Q. Jia, Unifying the Hydrogen Evolution and Oxidation Reactions Kinetics in Base by Identifying the Catalytic Roles of Hydroxyl-Water-Cation Adducts. Journal of the American Chemical Society 141, 3232-3239 (2019); published online EpubFeb 20 (10.1021/jacs.8b13228).

42. T. A. Richmond, T. T. Takahashi, R. Shimkhada, J. Bernsdorf, Engineered metal binding sites on green fluorescence protein. Biochemical and biophysical research communications 268, 462-465 (2000); published online EpubFeb 16 (10.1006/bbrc.1999.1244).

43. M. G. Romei, C.-Y. Lin, I. I. Mathews, S. G. Boxer, Electrostatic control of photoisomerization pathways in proteins. Science 367, 76-79 (2020).

44. T. Q. Yang, B. Peng, B. Q. Shan, Y. X. Zong, J. G. Jiang, P. Wu, K. Zhang, Origin of the Photoluminescence of Metal Nanoclusters: From Metal-Centered Emission to Ligand-Centered Emission. Nanomaterials 10, (2020); published online EpubFeb 4 
(10.3390/nano10020261).

45. T. Yang, S. Dai, S. Yang, L. Chen, P. Liu, K. Dong, J. Zhou, Y. Chen, H. Pan, S. Zhang, J. Chen, K. Zhang, P. Wu, J. Xu, Interfacial Clustering-Triggered Fluorescence-Phosphorescence Dual Solvoluminescence of Metal Nanoclusters. The journal of physical chemistry letters 8, 3980-3985 (2017); published online EpubSep 7 (10.1021/acs.jpclett.7b01736).

46. S. N. Baker, G. A. Baker, Luminescent carbon nanodots: emergent nanolights. Angewandte Chemie 49, 6726-6744 (2010); published online EpubSep 10 (10.1002/anie.200906623).

47. Z. Hu, B. J. Deibert, J. Li, Luminescent metal-organic frameworks for chemical sensing and explosive detection. Chemical Society reviews 43, 5815-5840 (2014); published online EpubAug 21 (10.1039/c4cs00010b).

48. D. Grandjean, E. Coutiño-Gonzalez, N. T. Cuong, E. Fron, W. Baekelant, S. Aghakhani, P. Schlexer, F. D'Acapito, D. Banerjee, M. B. J. Roeffaers, M. T. Nguyen, J. Hofkens, P. Lievens, Origin of the bright photoluminescence of few-atom silver clusters confined in LTA zeolites. Science 361, 686-690 (2018).

49. E. Coutino-Gonzalez, W. Baekelant, J. A. Steele, C. W. Kim, M. B. J. Roeffaers, J. Hofkens, Silver Clusters in Zeolites: From Self-Assembly to Ground-Breaking Luminescent Properties. Acc Chem Res 50, 2353-2361 (2017); published online EpubSep 19 (10.1021/acs.accounts.7b00295).

50. Z. Zhou, C. E. Hauke, B. Song, X. Li, P. J. Stang, T. R. Cook, Understanding the Effects of Coordination and Self-Assembly on an Emissive Phenothiazine. Journal of 
the American Chemical Society 141, 3717-3722 (2019); published online EpubFeb 27 (10.1021/jacs.9b00363).

51. S. Datta, M. L. Saha, P. J. Stang, Hierarchical Assemblies of Supramolecular Coordination Complexes. Acc Chem Res 51, $2047-2063$ (2018); published online EpubSep 18 (10.1021/acs.accounts.8b00233).

52. X. Yan, T. R. Cook, P. Wang, F. Huang, P. J. Stang, Highly emissive platinum(II) metallacages. Nature chemistry 7, 342-348 (2015); published online EpubApr (10.1038/nchem.2201).

53. Q. Zhou, B. Cao, C. Zhu, S. Xu, Y. Gong, W. Z. Yuan, Y. Zhang, Clustering-Triggered Emission of Nonconjugated Polyacrylonitrile. Small 12, 6586-6592 (2016); published online EpubDec (10.1002/smll.201601545).

54. D. A. Tomalia, B. Klajnert-Maculewicz, K. A. M. Johnson, H. F. Brinkman, A. Janaszewska, D. M. Hedstrand, Non-traditional intrinsic luminescence: inexplicable blue fluorescence observed for dendrimers, macromolecules and small molecular structures lacking traditional/conventional luminophores. Prog. Polym. Sci. 90, 35-117 (2019)10.1016/j.progpolymsci.2018.09.004).

55. Q. Fan, G. V. Biesold-McGee, J. Ma, Q. Xu, S. Pan, J. Peng, Z. Lin, Lead-Free Halide Perovskite Nanocrystals: Crystal Structures, Synthesis, Stabilities, and Optical Properties. Angewandte Chemie 59, 1030-1046 (2020); published online EpubJan 13 (10.1002/anie.201904862).

56. M. B. Faheem, B. Khan, C. Feng, M. U. Farooq, F. Raziq, Y. Xiao, Y. Li, All-Inorganic Perovskite Solar Cells: Energetics, Key Challenges, and Strategies toward 

Commercialization.
ACS
Energy
Letters
5 ,
290-320

(2019)10.1021/acsenergylett.9b02338).

57. M. A. Boles, D. Ling, T. Hyeon, D. V. Talapin, Erratum: The surface science of nanocrystals. Nature materials 15, 364 (2016); published online EpubMar (10.1038/nmat4578).

\section{TOC}
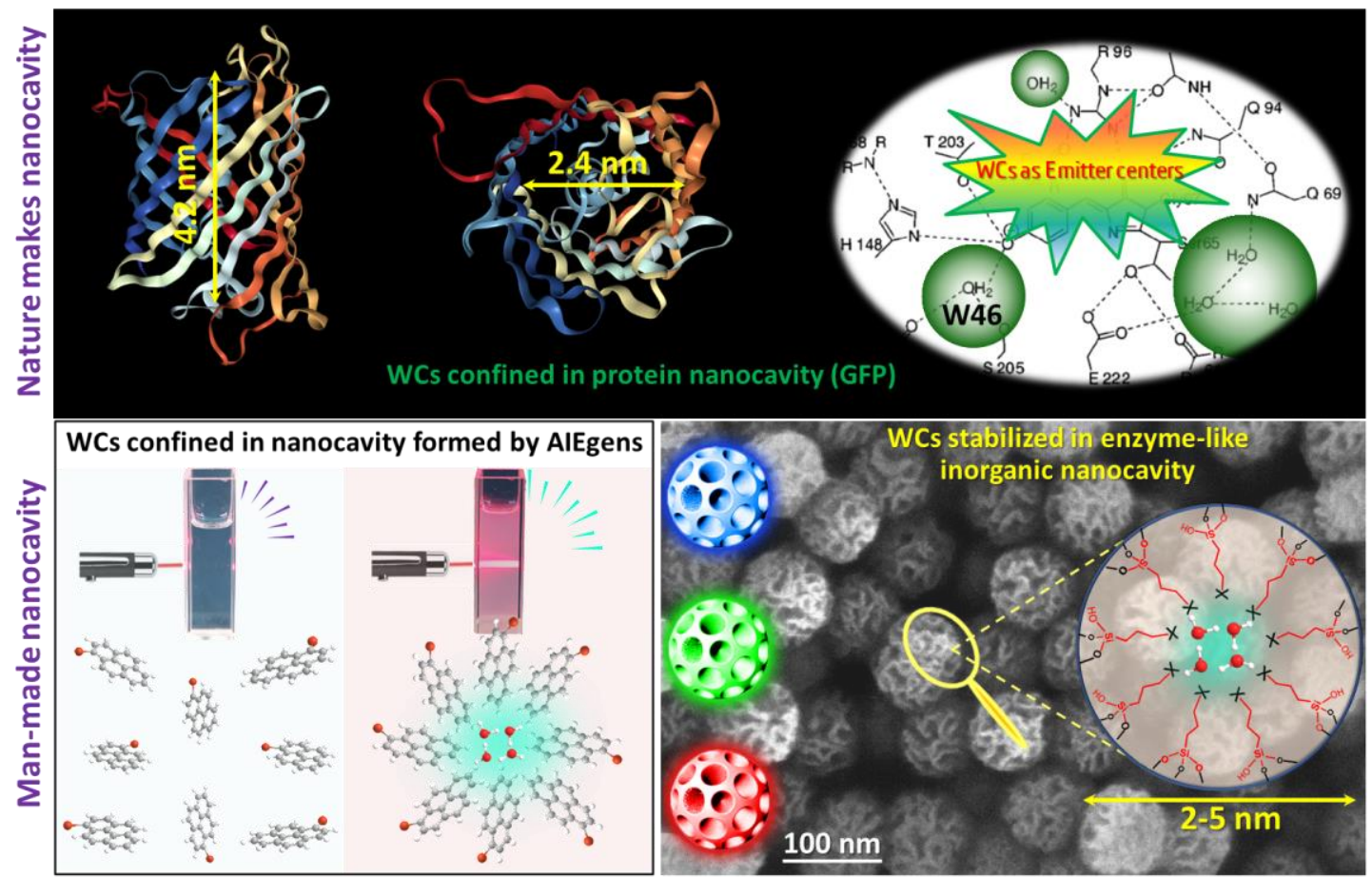

Intrinsically, free water molecules are colorless liquid. However, when the water clusters (WCs) are confined in the nanocavity, it shows strong photoluminescence (PL) with bright colors. 
